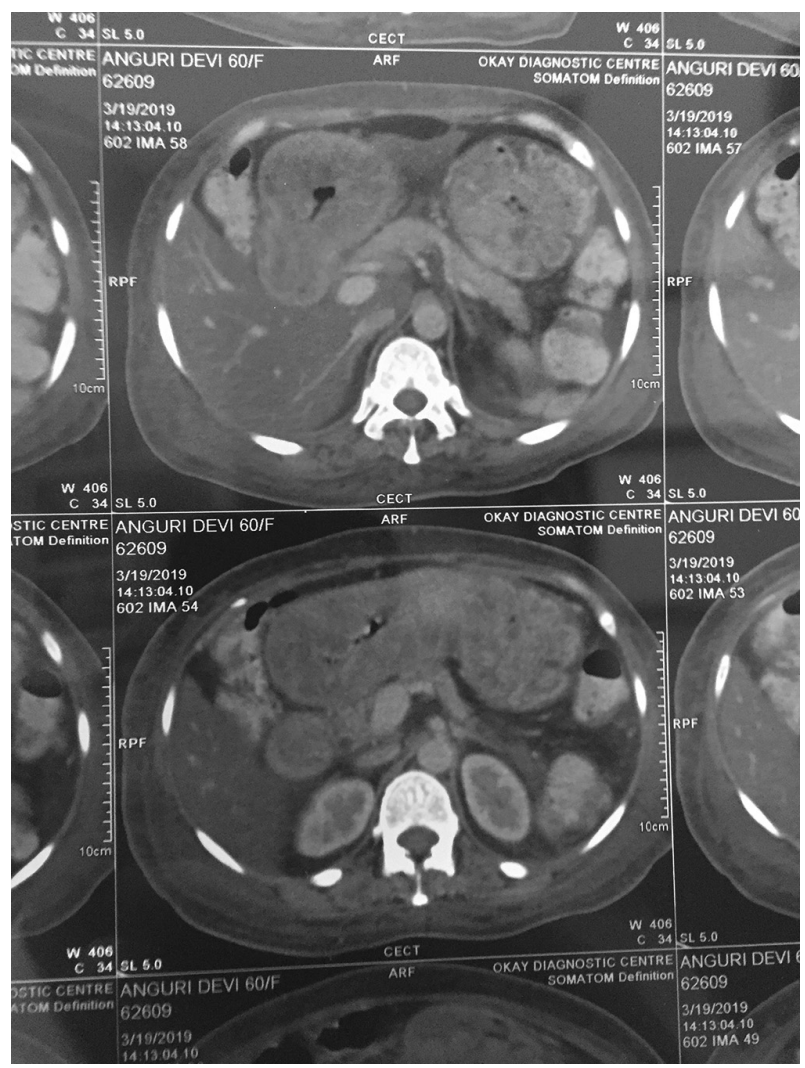

Abstract IDDF2019-ABS-0274 Figure 1 Gastroduodanl mass intramural air gastroduodenal intussusception

vomiting, abdominal distension, pain abdomen for 2 weeks. She had an earlier history of pain abdomen for which her UGI endoscopy was done which suggested polyp in the stomach, no biopsy taken. Now she has edema, ascites, distress for one week.

Methods The patient was worked up by blood tests including CBC, LFT, RFT. UGI endoscopy done, CECT abdomen done, ascitic fluid sent for microscopy, biochemical test and cytology. Exploratory laparotomy, abdominal doppler study is being planned by the time of submitting this abstract.

Results Ascitic fluid examination suggested high protein level, lymphocyte predominent fluid, negative malignant cytology. UGI endoscopy revealed gastric outlet obstruction, circumferential, soft, slimy, fungating growth in body and antrum of the stomach, with difficulty endoscopic biopsy was taken, tissue sent to the pathologist. CECT suggested thickened wall of the stomach in body and antrum, Gastroduodenal Intussusception, intramural air in bowel wall suggestive of bowel ischaemia. Complete blood count, Liver function test, renal function test was within normal range. The patient is admitted under GI surgeon for stabilization and definitive leiomyosarcoma treatment. Patient possibly has gastric carcinoma variant which can rarely cause intussusception like appearance after duodenal involvement, the air in gastric duodenal wall layers is rare possibly there is microleaks in the wall or due to rapid growth iscahemic changes, this area of GIT rarely have iscahemia being well vascularized. We will present complete details of histopathology and surgery in the meeting. Malignant ascites patient generally does not develop edema. We will do doppler study of the portal venous system to rule out Hepatic vein, portal vein or inferior venacava thrombosis. (figure 1)
Conclusions We hereby present a rare case of gastric mass with bowel ischaemia changes, Gastroduodenal Intussusception in an elderly lady presenting with ascites, edema, pain abdomen and vomiting. It can be gastric carcinoma, less likely gastric lymphoma or aggressive leiomyosarcoma. Ascites cytology needs to be repeated two times to exclude malignancy, while exploratory laparotomy will make things very clear, a larger specimen can be sent for histopathology. The patient will need some palliation for gastric outlet obstruction.

\section{IDDF2019-ABS-0276 A RARE CASE OF DUODENAL GIST WITH ENTEROCUTANEOUS FISTULA}

Supreeth Kumar Reddy Kunnuru*, Mayank Kumar Gurjar, Thirunavukkarasu Sampath, Varun Palanati, Rakesh Raja Reddy. Department of Surgical Gastroenterology, Narayana Medical College, India

\subsection{6/gutjnl-2019-IDDFabstracts.219}

Background Gist accounts for $0.1 \%$ to $0.3 \%$ of all gi malignancies and duodenal gist is even more rarer. This is an interesting case of duodenal gist with enterocutaneous fistula managed successfully.

Methods A 40-year-old female, operated for misdiagnosed appendicular lump 3 weeks back, presented to us with fecal fistula from scar site and mass in right hypochondrium extending to right iliac fossa region underwent all the investigations followed by exploratory laparotomy + enbloc resection of duodenal mass + right hemicolectomy + excision of fistulous tract + duodenojejunostomy was done.

Results Post surgery period was uneventful

Biopsy: spindle cell type gist, margins free of tumor, mitotic index - 5/50 HPF, high grade, pT4N0Mx

The patient received imatinib therapy post operatively Conclusions This is a rare case of large duodenal gist infiltrating hepatic flexure and ascending colon with enterocutaneous fistula managed successfully with wedge resection of duodenum avoiding Whipple procedure. Gist when enblock resection done with negative margins without any metastasis, show good prognosis post operatively

\section{IDDF2019-ABS-0282 TICKING TIMEBOMBS DUE TO PANCREATITIS}

${ }^{1}$ Tripuraneni Venkata Aditya Chowdary ${ }^{*},{ }^{2} \mathrm{CH}$ Madhusudhan, ${ }^{2} \mathrm{R}$ Pratap Reddy. ${ }^{1}$ Gleneagles Global Hospital, India; ${ }^{2}$ Osmania Medical College, India

\subsection{6/gutjnl-2019-IDDFabstracts.220}

Background Visceral arterial pseudo aneurysms are rare and usually involve the splenic artery. They mostly occur in the background of pancreatitis, with trauma being the second most common cause. The detection of the pseudoaneurysm has increased with our lowered threshold of using high-resolution imaging (CT/MRI/Doppler). Most of them are symptomatic and present a dilemma for management. The aim of the study was to identify all patients with abdominal visceral arterial pseudo aneurysms, analyze their clinic-pathological features, management and outcome.

Methods The study was conducted in the Surgical Gastroenterology Department of Osmania Medical College and Hospital. All patients who were diagnosed to have a splenic artery pseudo aneurysm from the January 2012 to July 2016 were included in the study 
Results A total of 15 cases were identified, splenic artery was the origin in thirteen and gastroduodenal artery in two. All patients were male, pancreatitis accounted for 14 (93.33\%) and trauma for $1(6.66 \%)$. All patients were symptomatic with abdominal pain (80\%), GI bleed (66.66\%) and fall in hemoglobin $(66.66 \%)$ being the common symptoms. CECT with vascular reconstruction was the best investigatory modality to identify them. Angio-embolization was used in two patients with good outcomes. Percutaneous thrombin was used in one patient but unsuccessful. Surgery was used in 13 patients (distal pancreaticosplenectomy - 8, transpesudocystic ligation - 3 and direct aneurysm excision - 1).

Conclusions Visceral arterial pseudo aneurysms are not as rare as previously thought and the incidence is rising as our threshold for imaging is falling. Their management is multidisciplinary and depends on the resources available. All of them are invariably symptomatic and require intervention of some sort for a permanent control. The threshold to offer surgery should be low as the morbidity (26.66\%) and mortality $(6.66 \%)$ are relatively low.

\section{IDDF2019-ABS-0283 LAPAROSCOPIC-ASSISTED TRANSGASTRIC ERCP IN PATIENTS WITH GASTRIC BYPASS: A SINGLE CENTRE EXPERIENCE}

Tripuraneni Venkata Aditya Chowdary*,Lakshmi Kona, Rakesh Kumar Adi. Gleneagles Global Hospital, India

\subsection{6/gutjnl-2019-IDDFabstracts.221}

Background The management of patients who develop common bile duct calculi post gastric bypass is complicated as transoral access to the biliary tree is not possible. Majority of the patients may require a lap/open cbd exploration. We here describe our technique of accessing the cbd laparoscopically via the ampulla of vater.

Methods Between January 2014 and January 201912 patients developed CBD calculi. $\underline{7}$ of them had undergone Roux en $y$ gastric bypass (RYGB) and 5 of them had undergone mini gastric bypass (MGB).

A combined laparoscopic-endoscopic approach was attempted. A small gastrotomy with a purse-string suture was performed on the anterior wall on remanant stomach. A side viewing scope was introduced through a $15 \mathrm{~mm}$ trocar on the upper left quadrant and through the gastrotomy. The duodenum was occluded to prevent air passage and small bowel distension. Endoscopic sphincterotomy and stone extraction were carried out according to standard techniques. Occlusion cholangiogram confirmed CBD clearance. There was no procedure-related complication, and the patients were discharged on the second postoperative day. The patient is doing well at 6months follow up.

Results The detection of CBD calculi was on an average after 3 years post gastric bypass. All patients were managed as per the described technique and the mean hospital stay was 3 days. Seven patients underwent simultaneous cholecystectomy. Two patients had cholecystectomy more than 2 years previously. Our technique involves access to the bypassed stomach via a laparoscopically placed $15 \mathrm{~mm}$ port. We observed no major complications. No minor complications except, port site infection for one patient was observed in our series.
Conclusions The formation of gallstones post gastric bypass is a known entity and they can migrate into the CBD. Management of these presents a unique dilemma. LAERCP (Laproscopic ERCP) is a reliable option for common bile duct clearance; our technique of LAERCP is technically simple and associated with low complication rate, making it appealing to surgeons trained in laparoscopy.

\section{IDDF2019-ABS-0284 BRUNNER'S GLAND HYPERPLASIA: A CASE SERIES}

Nara Bharat Kumar*, Tripuraneni Venkata Aditya Chowdary. Gleneagles Global Hospital, India

10.1136/gutjnl-2019-IDDFabstracts.222

Background Brunner's glands are exocrine glands located in proximal duodenum. Except for its association with duodenal ulcers, Brunner's gland hyperplasia has no clinical significance and mostly asymptomatic. Rarely one or few hyperplastic Brunner's glands may form a mass lesion large enough to cause obstructive symptoms, epigastric pain, or upper GI bleeding. It is important to differentiate them from malignancy. Here we are presenting two cases of Brunner's gland hyperplasia which were difficult to distinguish from neoplasia

Methods All patients who on pathology were diagnosed to have Brunner's gland hyperplasia at our center were studied.

Results Case 1, a 61-year old female was presented with history intermittent epigastric fullness associated non-bilious vomiting for $\underline{6}$ months. Endoscopy - large bossellated exophytic lesion in of D1. CECT $48 \times 31 \times 33 \mathrm{~cm}$ well defined minimally enhancing lesion in the medial wall in the D1 causing luminal narrowing. A EUS guided FNAB showed Brunner's gland hyperplasia.

She underwent Laparoscopic Limited Resection of Antrum and 1st part of duodenum with Anterior Ante-colic loop Gastrojejunostomy. Her histopathology revealed burner's gland hyperplasia.

Case 2, a 31-year old male presented with epigastric pain, nonbilious vomiting, anorexia for three months with a weight loss of $15 \mathrm{kgs}$.

Endoscopy- duodenal narrowing with nodular mass in the periampullary area. CECT- $55 * 42 * 35 \mathrm{~mm}$ heterotropic mass arising from the second part of the duodenum with indistinct planes with the head of the pancreas. Surgery - Whipple's operation and recovered well post operatively. His histopathology revealed Brunner's gland hyperplasia.

Conclusions Primary duodenal tumors are extremely rare, accounting for less than $1 \%$ among all Gl tumors and Brunner's gland adenoma has an incidence of less than $0.01 \%$

Most patients with Brunner's gland adenoma are asymptomatic or have complaints such as nausea, bloating, or vague abdominal pain. EUS-FNAC may remain inconclusive and surgical excision is required to confirm the diagnosis and to relieve symptoms of obstruction and bleeding. Laparoscopic excision can be done if there is preop. Diagnosis of benign pathology as here in case 1 , however- a Whipple procedure may only an option when it is indistinguished from malignancy. There have been no reports of recurrence after either endoscopic or surgical resection. They are benign and have a good prognosis 